\title{
The Sleeve Gastrectomy and How and Why it can Fail?
}

\author{
Noah J Switzer ${ }^{1}$ and Shahzeer Karmali1,2* \\ ${ }^{1}$ Department of Surgery, University of Alberta, Edmonton, Alberta, Canada \\ ${ }^{2}$ Center for the Advancement of Minimally Invasive Surgery (CAMIS), Royal Alexandria Hospital, Edmonton, Alberta, Canada
}

\begin{abstract}
Long-term weight regain is a fearedcomplicationof restrictive bariatric operation. The Sleeve Gastrectomy (SG) is still in its early stages as a primary bariatric surgery and long-term data about its efficacy remains limited. From the long term studies available it seems that approximately one-fifth of SG patients might be at risk for long-term weight regain and about $5-10 \%$ of total SG patients will require surgical management forit. The possible mechanism behind this weight regain is slowly being addressed. Patient noncompliance with dietary and lifestyle regimens is the most practical factor that needs to be considered and can be prevented with a multidisciplinary team. Long-term gastric pouch dilatation and gut hormone modulation are other theories that have been proposed to explain this weight regain. Successful management strategies to combat weight recidivism include revisional bariatric surgery, performing a resleeve gastrectomy or the addition of an adjustable band in the primary banded sleeve gastrectomy. However, the safety of revisional bariatric surgery is a concern and should be performed only by an experienced bariatric surgeon. It remains that as the SG continues to grow as a popular choice for the management of morbid obesity, more concrete long term information will become available to address the how and why weight regain occurs.
\end{abstract}

Keywords: Sleeve gastrectomy; SG patients

\section{Introduction}

Weight recidivism after primary bariatric procedures is an important issue and concern for many bariatric patients. Regardless of the specific type of bariatric surgery, long term weight regain will occur to a small but significant proportion of patients [1]. Weight regain is especially prevalent in the restrictive bariatric procedures, operations that limit the amount of oral intake compared to malabsorptive procedures [2]. The Sleeve Gastrectomy (SG) is still a newer primary bariatric surgery, formerly being the first step in a staged procedure for higher risk bariatric patients [3]. SG, considered mainly a restrictive procedure, removes the greater curvature of the stomach, thereby reducing the size of the stomach to $60-80 \mathrm{~mL}$ in capacity and modifying its shape to be more tubular in nature $[4,5]$. It has gained popularity as a standalone bariatric surgery due to its relative operational simplicity, lack of foreign body implantation and with an undisrupted gastrointestinal tract, it lacksthe dumping syndrome and nutrient deficiencies seen with the malabsorptive operations [6-8]. However due to its relative infancy, data on the long term complication of weight recidivism with it being a primary procedure is still unknown [7,9]. Furthermore, anypast data on significant weight regain following SG, as a staged procedure, was not highlighted as a second, planned surgery was still being scheduled to compensate [9]. In this paper, we review the literature for weight regain following the Sleeve Gastrectomy, theorize why this complication can occur and recommend strategy options for dealing with this feared complication.

\section{Discussion}

Weight regain to an extent should be expected after all bariatric surgeries, however significant weight gain, described as an increase in body weight of $10 \mathrm{~kg}$ from nadir, can mean a failure of the procedure $[9,10]$. Non-modifiable risk factors for failure include age $>40$ and preoperative $\mathrm{BMI}>50$ [11]. Restrictive procedures like the SG, which lack an intestinal bypass, are especially at risk [8]. In one of the few longer term case series reported on SG, Bohdjalian et al. reported a weight regain incidence of $19.2 \%$ [9]. This weight regain was first recognized by the end of the second post-operative year [9]. Interestingly, D'hondt et al. reported a trend of slight weight regain annuallyafter SG through observing continued decreasing Excess Weight Loss (EWL) at annual intervals; at 1 year follow up patients had a median EWL of $81.5 \%$ which dropped to $55.9 \%$ after 6 years [12]. Different studies report varying numbers forthe percentage of primary SG patients who will require an unscheduled secondary operation to account for insufficient weight loss or weight regain but it appears that the number is somewhere between $5-10 \%[1,2,13]$.

An important and devastating consequence of this weight regain is the potential recurrence of obesity related comorbidities such as type 2 Diabetes mellitus. Jimenez et al. found that insufficient weight loss or weight regain were independent long-term predictors of recurrence of diabetes following SG [14]. This lends credence to the importance of recognizing and treating weight re-gains patients.

\section{Theories to explain weight recidivism following SG}

The most practical and first consideration that needs to be addressed in a patient presenting with weight recidivism is adherence to dietary and lifestyle regimens as outlined by the multidisciplinary team [15]. Modifiable risk factors for failure after SG include a lack of physical activity, a lack of change of nutritional behavior, and a lack of follow-up [11]. The importance of proper nutritional counseling after bariatric surgery has been supported by the literature. A significant positive association has been shown between patient vigilance with maintaining food records and weight loss; while on the other hand, there is a significant negative association between nutritional counseling attendance and weight gain [16,17]. Furthermore, Himpens et al. showed that craving for sweet eating tends to increase the further out from SG surgery [18]. SG is not a malabsorptive procedure therefore any high calorie junk foods will be readily absorbed, leading to potential significant weight gain. This patient "cheating" must be an important first consideration when assessing for contributing factors leading

*Corresponding author: Shahzeer Karmali, Center for the Advancement of Minimally Invasive Surgery (CAMIS), Royal Alexandra Hospital, 10240 Kingsway, Edmonton, Alberta, T5H 3V9, Canada, Tel: (780) 735-6650; Fax: (780) 735-6652; E-mail: shahzeer@ualberta.ca

Received January 03, 2013; Accepted February 11, 2014; Published February 20, 2014

Citation: Switzer NJ, Karmali S (2014) The Sleeve Gastrectomy and How and Why it can Fail? Surgery Curr Res 4: 180. doi:10.4172/2161-1076.1000180

Copyright: (c) 2014 Switzer NJ, et al. This is an open-access article distributed under the terms of the Creative Commons Attribution License, which permits unrestricted use, distribution, and reproduction in any medium, provided the original author and source are credited. 
to SG failure [19]. Psychological factors also need to be addressed in a patient first presenting with weight re-gain, as there is a marked correlation between weight-regain and psychiatric co-morbidities [20]. The weight wise clinic in Edmonton, Alberta Canada, is a model for a multidisciplinary clinic where patients are followed post-operatively to ensure maximum compliance and behavioral modification [4].

Long-term gastric pouch dilatation is the primary theorized explanation for weight regain in the post-operative period following SG. Patient noncompliance with diet regimenscan lead to mechanical stretching of the gastric pouchdue to consistent intake of larger meals [15]. Patients need to comply with strict dietary habits to ensure minimal stretching of the gastric pouch. However, operator technical error at the initial operation can also lead to this result. Not recognizing the presence of a hiatal hernia, the incomplete removal of the gastric fundusorthe creation of an excessively large gastric pouch due to either missed posterior gastric folds or calibration with an inappropriately large boogie, will all lead to long-term pouch dilatation. Both smaller volume gastrectomies (under $500 \mathrm{cc}$ ) at the initial surgery and high residual gastric volume post-operatively (over $225 \mathrm{cc}$ ) were associated with failure of SG, emphasizing the importance of restriction $[11,12]$.

The incidence of gastric dilatation leading to attributed weight regains remains unknown and the literature inconsistent [21]. A case report by Gagner et al., reported the presentation of poor weight loss in a patient following a staged procedure combining sleeve gastrectomy and biliopancreatic diversion with duodenal switch (BDP-DS) [22]. Initially the patient experienced significant weight loss (80\% of Excess Weight Loss (EWL)) but after $>2$ years post-op she returned with weight regain. Upper GI series demonstrated a markedly dilated gastric pouch, which the authors hypothesized as the culprit for the weight gain [22]. In a more recent prospective study, Langer et al. performed upper GI series at 1 year follow in post-op SG patients and found only 1 out of 14 patients to fit the criteria for gastric dilation and yet surprisingly that patient still achieved and maintained adequate EWL [6]. Interestingly, in another Langer et al. study, weight regain in patientswho required revisional bariatric surgery was not correlated with substantial sleeve dilation [2].

Overall, the tendency for long-term gastric pouch dilatation following SG, independent of operator technical error, remains unproven. However, it appears that patients are potentially most at risk for weight recidivism via gastric dilatation at 2 years and beyond following their initial SG $[6,9,22]$. Still, it is clear that additional longterm studies should be devoted to further clarify this issue.

Another possible explanation for the weight regain in the months to years following SG could be explained by the regulation of gut hormones, namely ghrelin, following bariatric surgery. Ghrelin is an appetitestimulating hormone, which is produced mainly in the gastric fundus and body $[9,23]$. The SG involves the removal of the gastric fundus and as such, leads to early and maintained reduced ghrelin levels in most successful patients $[9,24]$. A recent systematic review by Anderson et al. reported that ghrelin levels were significantly reduced at 3, 6 and 12 months after laparoscopic SG [24]. It seems that unsuccessful weight regain patients might have compensatory increases in long term plasma ghrelin levels, leading to increasing appetite stimulation, which might explain the SG failure [9]. 75\% of SG patients were reported to have a loss of feeling of hunger at 1 year following SG, but this number dropped significantly to $46.7 \%$ at 3 year follow-up [18]. Still, there remains inconsistency in the literature as to the effect of ghrelin levels following bariatric surgery and its implications on weight loss or gain, and more research needs to be done to be comfortable making a conclusion [24].

\section{Strategies for management of weight recidivism}

Three main treatment possibilities exist for management of weight recidivism patients: Conversion to a malabsorptive bariatric procedure, a redo "Re-sleeve gastrectomy" or adding an adjustable gastric band $[7,8,25]$. Conversion from failed SG to another bariatric procedure, usually to either Roux en-Y Gastric Bypass (RYGB) or BPD-DS remains a viable treatment for weight regain. Historically, SG was originally the first step in a scheduled two-step operation, where patients experienced significant additional EWL following conversion to either duodenal switch or RYGB $[7,10]$. It is therefore an easier transition to convert it to a second unscheduled bariatric procedure for weight loss failure [8]. Adding malabsorption to the already restrictive $S G$ has been proven to be an effective means for further weight loss [2]. The main concern with revisional bariatric surgery is that the complication rates are significantly higher than in the primary procedure [26]. In addition, Langer et al., however, urge caution stating that "weight regainer" patients after SG might be more susceptible to be "weight regainers" following conversion. The safety of revisional bariatric surgery is controversial in the literature, with complication rates of converting to RYGB being considerable and varied (ranging from $0-47 \%$ ), yet still RYGB is considered the safer option compared to BPD-DS [27]. Therefore, due to this high risk patient population and the challenges of operating on altered and scarred anatomy, revisional bariatric surgery should be performed by experienced bariatric surgeons at a tertiary care hospital to ensure maximum patient safety and procedural success, this type of model has been demonstrated to be quite effective with acceptable patient morbidity $[15,26,28]$. The Royal Alexandra Hospital in Edmonton, Alberta, Canada, recently opened dedicated weight recidivism after bariatric surgery clinic, specific for pre-operatively patient selection, in order to determine which patients would benefit from revisional surgery. This inevitably will become the model clinic for tertiary bariatric centers across the world. Repeating a second SG, named a "re-sleeve" gastrectomy, is another option. This procedure is usually indicated in the event of insufficient weight loss or weight regain due to isolated gastric dilatation $[8,19,29]$. Iannelli et al. reported EWL of $46.5 \%$ in their patients with a re-do SG, in a described "technically relatively easy" operation. The operator must take into consideration important surgical revision complications when advising their patient for this option, which include increased risk of leakage at the Angle of His and risk of injury to the splenic vein [7]. It is important to remember, however, that repeating a SG does not protect the patient from are occurrence of gastric dilation and long-term weight regains [7].

The primary banded sleeve gastrectomy places a band below the gastroesophageal junction in addition to the SG in an attempt to further weight loss [30]. Greenstein et al. first introduced this idea of adding an adjustable gastric band to SG patients to increase gastric restriction ENREF $[24,25]$. The authors state that in SG patients where primary restriction has failed, a band can be a successful option that avoids the complications associated with revisional bariatric surgery like staple line bleeds and leaks [25]. It can also be used as preventative means to avoid SG failure in the first place [30].

\section{Conclusion}

Weight regains following the Sleeve Gastrectomy is a concern. Due to its relative infancy, there exists limited information on long-term outcomes following this procedure. The role of gastric dilatation and long term circulating ghrelin levels in causing weight regain needs to be further clarified. The management options for significant weight regain are emerging and include conversion to a malabsorptive procedure, 
Citation: Switzer NJ, Karmali S (2014) The Sleeve Gastrectomy and How and Why it can Fail? Surgery Curr Res 4: 180. doi:10.4172/21611076.1000180

performing a re-sleeve or adding an adjustable band. The safety of these procedures remains concerning and involving an experienced bariatric surgeon at a tertiary care hospital is of the utmost importance.

\section{References}

1. Gautier T, Sarcher T, Contival N, Le Roux Y, Alves A (2013) Indications and mid-term results of conversion from sleeve gastrectomy to Roux-en-Y gastric bypass. Obes Surg 23: 212-215.

2. Langer FB, Bohdjalian A, Shakeri-Leidenmühler S, Schoppmann SF, Zacherl J, et al. (2010) Conversion from sleeve gastrectomy to Roux-en-Y gastric bypass-indications and outcome. Obes Surg 20: 835-840.

3. Almogy G, Crookes PF, Anthone GJ (2004) Longitudinal gastrectomy as a treatment for the high-risk super-obese patient. Obes Surg 14: 492-497.

4. Gill RS, Switzer N, Driedger M, Shi X, Vizhul A, et al. (2012) Laparoscopic sleeve gastrectomy with staple line buttress reinforcement in 116 consecutive morbidly obese patients. Obes Surg 22: 560-564.

5. Braghetto I, Korn O, Valladares H, Gutiérrez L, Csendes A, et al. (2007) Laparoscopic sleeve gastrectomy: surgical technique, indications and clinical results. Obes Surg 17: 1442-1450.

6. Langer FB, Bohdjalian A, Felberbauer FX, Fleischmann E, Reza Hoda MA, et al. (2006) Does gastric dilatation limit the success of sleeve gastrectomy as a sole operation for morbid obesity? Obes Surg 16: 166-171.

7. Iannelli A, Schneck AS, Noel P, Ben Amor I, Krawczykowski D, et al. (2011) Re-sleeve gastrectomy for failed laparoscopic sleeve gastrectomy: a feasibility study. Obes Surg 21: 832-835.

8. Baltasar A, Serra C, Perez N, Bou R (2006) Re-sleeve gastrectomy. ObesSurg16:1535-1538.

9. Bohdjalian A, Langer FB, Shakeri-Leidenmühler S, Gfrerer L, Ludvik B, et al. (2010) Sleeve gastrectomy as sole and definitive bariatric procedure: 5-year results for weight loss and ghrelin. Obes Surg 20: 535-540.

10. Langer FB, Shakeri-Leidenmühler S, Bohdjalian A, Schoppmann S, Zacher J, et al. (2010) Strategies for weight regain after sleeve gastrectomy. Surg Laparosc Endosc Percutan Tech 20: 159-161.

11. Deguines JB, Verhaeghe $P$, Yzet $T$, Robert $B$, et al. (2006) Is the residual gastric volume after laparoscopic sleeve gastrectomy an objective criterion for adapting the treatment strategy after failure? Surgery for obesity and related diseases: official journal of the American Society for Bariatric Surgery 9: 660-666.

12. D'Hondt M, Vanneste S, Pottel H, Devriendt D, Van Rooy F, et al. (2011) Laparoscopic sleeve gastrectomy as a single-stage procedure for the treatment of morbid obesity and the resulting quality of life, resolution of comorbidities, food tolerance, and 6-year weight loss Surg Endosc 25: 2498-2504.

13. Weiner RA, Theodoridou S, Weiner S (2011) Failure of laparoscopic sleeve gastrectomy--further procedure? Obes Facts 4 Suppl 1: 42-46.

14. Jiménez A, Casamitjana R, Flores L, Viaplana J, Corcelles R, et al. (2012) Long term effects of sleeve gastrectomy and Roux-en-Y gastric bypass surgery on type 2 diabetes mellitus in morbidly obese subjects. Ann Surg 256: 1023-1029.
15. Zundel N, Hernandez JD (2010) Revisional surgery after restrictive procedures for morbid obesity. Surg Laparosc Endosc Percutan Tech 20: 338-343.

16. Odom J, Zalesin KC, Washington TL, Miller WW, Hakmeh B, et al. (2010) Behavioral predictors of weight regain after bariatric surgery. Obes Surg 20 349-356.

17. Freire RH, Borges MC, Alvarez-LeiteJl, Toulson Davisson Correia MI (2012) Food quality, physical activity, and nutritional follow-up as determinant of weight regain after roux-en-y gastric bypass. Nutrition 28:53-58

18. Himpens J, Dapri G, Cadière GB (2006) A prospective randomized study between laparoscopic gastric banding and laparoscopic isolated sleeve gastrectomy: results after 1 and 3 years. Obes Surg 16: 1450-1456.

19. Dapri G, Cadiere GB, Himpens J (2011) Laparoscopic repeat sleeve gastrectomy versus duodenal switch after isolated sleeve gastrectomy for obesity. Surg Obes Relat Dis 7: 38-43.

20. Rutledge T, Groesz LM, Savu M (2011) Psychiatric factors and weight loss patterns following gastric bypass surgery in a veteran population. Obes Surg 21: 29-35.

21. Weiner RA, Weiner S, Pomhoff I, Jacobi C, Makarewicz W, et al. (2007) Laparoscopic sleeve gastrectomy--influence of sleeve size and resected gastric volume. ObesSurg 17: 1297-1305.

22. Gagner M, Rogula T (2003) Laparoscopic reoperative sleeve gastrectomy for poor weight loss after biliopancreatic diversion with duodenal switch. Obes Surg 13: 649-654.

23. Ashrafian H, le Roux CW (2009) Metabolic surgery and gut hormones - a review of bariatric entero-humoral modulation. PhysiolBehav 97: 620-631.

24. Anderson B, Switzer NJ, Almamar A, Shi X, Birch DW, et al. (2013) The impact of laparoscopic sleeve gastrectomy on plasma ghrelin levels: a systematic review. ObesSurg 23: 1476-1480.

25. Greenstein AJ, Jacob BP (2008) Placement of a laparoscopic adjustable gastric band after failed sleeve gastrectomy. Surg Obes Relat Dis 4: 556-558.

26. Shimizu H, Annaberdyev S, Motamarry I, Kroh M, Schauer PR, et al. (2013) Revisional bariatric surgery for unsuccessful weight loss and complications. Obes Surg 23: 1766-1773.

27. Deylgat B, D'Hondt M, Pottel H, Vansteenkiste F, Van Rooy F, et al. (2012) Indications, safety, and feasibility of conversion of failed bariatric surgery to Roux-en-Y gastric bypass: a retrospective comparative study with primary laparoscopic Roux-en-Y gastric bypass. Surg Endosc 26: 1997-2002.

28. Stefanidis D, Malireddy K, Kuwada T, Phillips R, Zoog E, et al. (2013) Revisiona bariatric surgery: perioperative morbidity is determined by type of procedure. Surg Endosc 27: 4504-4510.

29. Catheline JM, Fysekidis M, Dbouk R, Boschetto A, Bihan H, et al. (2012) Weight loss after sleeve gastrectomy in super superobesity. J Obes 2012: 959260

30. Alexander JW, Martin Hawver LR, Goodman HR (2009) Banded sleeve gastrectomy--initial experience. Obes Surg 19: 1591-1596. 